\title{
Methylation and Expression of Retinoblastoma and Transforming Growth Factor- $\beta 1$ Genes in Epstein-Barr Virus-Associated and -Negative Gastric Carcinomas
}

\author{
Xia Liu, ${ }^{1}$ Xiuming Tang, ${ }^{2}$ Shaoyan Zhang, ${ }^{3}$ Yun Wang, ${ }^{1}$ Xiaofeng Wang, \\ Chengquan Zhao, ${ }^{4}$ and Bing Luo ${ }^{1}$ \\ ${ }^{1}$ Department of Medical Microbiology, Qingdao University Medical College, 38 Dengzhou Road, Qingdao 266021, China \\ ${ }^{2}$ Department of Central Laboratory, The Affiliated Hospital of Qingdao University Medical College, 19 Jiangsu Road, \\ Qingdao 266003, China \\ ${ }^{3}$ Department of Laboratory, The Affiliated Hospital of Qingdao University Medical College, \\ Qingdao University, 19 Jiangsu Road, Qingdao 266003, China \\ ${ }^{4}$ Department of Pathology, University of Pittsburgh Medical Center, Pittsburgh PA 15213, USA
}

Correspondence should be addressed to Bing Luo, qdluobing@yahoo.com

Received 25 May 2012; Revised 8 August 2012; Accepted 13 August 2012

Academic Editor: A. Castells

Copyright ( 2012 Xia Liu et al. This is an open access article distributed under the Creative Commons Attribution License, which permits unrestricted use, distribution, and reproduction in any medium, provided the original work is properly cited.

\begin{abstract}
Background. Retinoblastoma $(R B)$ and transforming growth factor- $\beta 1$ (TGF- $\beta 1)$ are important tumor-related factors. Methods. A series of $30 \mathrm{EBV}$-associated gastric carcinoma (EBVaGC) and 38 matched EBV-negative gastric carcinoma (EBVnGC) tissues were examined for the promoter methylation of $R B$ by methylation-specific PCR (MSP) method. The expression of RB and TGF$\beta 1$ in gastric carcinoma tissues was detected by immunohistochemistry. Results. The methylation rate of $R B$ gene in EBVaGC and EBVnGC was $80.0 \%(24 / 30)$ and $50.0 \%$ (19/38), respectively. The difference of $R B$ methylation rate between EBVaGC and EBVnGC was significant $\left(\chi^{2}=6.490, P=0.011\right)$. There was no significant difference for $R B$ expression between EBVaGC (43.3\%, $13 / 30)$ and EBVnGC $(63.2 \%, 24 / 38)$, and also for TGF- $\beta 1$ between EBVaGC $(56.7 \%, 17 / 30)$ and EBVnGC $(63.2 \%, 24 / 38) . R B$ methylation was not reversely correlated with $R B$ expression in gastric carcinoma tissues $\left(\chi^{2}=2.943, P=0.086, r=0.208\right)$. $R B$ methylation, loss expression of $R B$, and TGF- $\beta 1$ expression were significantly associated with tumor invasion and lymph node metastasis $(P<0.05)$, but was not associated with sex, age, histological subtype (differentiation status) and tumor location. Conclusions. Methylation of $R B$ is a common event in gastric carcinomas and EBV induces methylation of $R B$ in EBVaGC, which may contribute to the development of gastric carcinomas. EBV has no significant effect on induction of TGF- $\beta 1$ expression. Detection of $R B$ methylation, $R B$ expression, and TGF- $\beta 1$ expression may be helpful to judge the status of tumor invasion and lymph node metastasis in gastric carcinomas.
\end{abstract}

\section{Introduction}

Gastric carcinoma is the second leading cause of cancerrelated death worldwide [1]. Epstein-Barr virus (EBV) is a tumor-related herpes virus associated with the transformation of various types of cells, such as lymphoid, dendritic, smooth muscle, and epithelial cells [2]. EBVassociated gastric carcinoma (EBVaGC) is characterized by the monoclonal growth of EBV-infected epithelial cells, and the entity was recognized by Imai in 1994 [3]. EBVaGC is distributed worldwide with an annual incidence of more than 90,000 patients (10\% of total gastric carcinoma (GC))
[4]. Following infection, EBV remains in a latent state in EBVaGC, which is classified as latency I. Compared with EBV-negative gastric carcinoma (EBVnGC), EBVaGC has unique clinical and pathological features, such as younger age of incidence, high incidence in men than in women, and more diffuse than intestinal types [5-7], suggestive of a particular oncogenic mechanism of EBVaGC.

Epigenetic alterations, including methylation of $\mathrm{CpG}$ dinucletides in promoters and changes in chromatin structure, can affect gene expression without modifying the underlying in genetic sequences. Aberrant methylation of promoters in tumor-related genes is now regarded as one 
of the major mechanisms in the development of gastric carcinoma [8]. Tumor-related genes p16, p14, E-cadherin, PTEN (phosphatase and tensin homolog deleted on chromosome ten), RASSF1A (Ras association domain family 1A), GSTP1 (Glutathione S-transferase pi 1), MGMT (O (6)-methylguanine-DNA-methyltransferase), and MINT2 (Munc18-1-interacting protein 2) are hypermethylated in EBVaGC [9-11], suggesting that EBV-related aberrant methylation may play an important role in development of EBVaGC.

Retinoblastoma $(R B)$ and transforming growth factor- $\beta 1$ (TGF- $\beta 1$ ) are important regulatory factors in cell growth and differentiation, whose abnormal transcription or expression are closely associated with tumor occurrence and development. $R B$ was the first successfully cloned human tumor suppressor gene (TSG). Its inactivation may result in cell proliferation leading to tumorigenesis [12]. TGF- $\beta 1$ is a multifunctional cytokine and triggers an intracellular signal transduction protein to regulate numerous developmental and homoeostatic processes via regulation of gene induction. It plays a dual regulatory role in cell proliferation and differentiation. In the early stage of cancer, TGF- $\beta 1$ can inhibit cell proliferation through arrest in the G1 phase and be regarded as a tumor suppressor; in the late stages, $T G F-\beta 1$ becomes a tumor promoting factor by stimulating angiogenesis, cell spread, immune suppression, and synthesis of extracellular matrix [13-16]. It has been reported that EBV latent membrane protein 1 (LMP1) has a resistant to the TGF- $\beta 1$-mediated growth inhibition in EBV-positive gastric carcinoma cell lines (GT38 and GT39) and indicated that TGF- $\beta 1$ may be a key factor for EBV reactivation and selective growth of EBV-infected epithelial cells in vivo [17, 18]. However, the LMP1 expression is absent in EBVaGC tissues [19]. The identified role of TGF- $\beta 1$ in EBVaGC has not been understood well and needs further research. The absence of $R B$ expression and overexpression of TGF- $\beta 1$ have been found in gastric carcinomas [20,21], and the mutations and methylation of $R B$ gene in gastric carcinomas were also reported in the literature [22, 23]. Mukherjee et al. [24] found TGF- $\beta 1$ treatment in late G(1) acutely blocks S-phase entry, this acute block by requiring the function of $R B$ and loss of RB abrogates late-G(1) arrest by TGF- $\beta 1$, suggesting a novel role for $R B$ in mediating this effect of TGF- $\beta 1$ late$G(1)$ arrest through direct interaction with and control of the MCM helicase. However, there is no report about the expression and promoter methylation status of $R B$ and TGF$\beta 1$ in EBVaGC and EBVnGC to our knowledge. In this study, we examined $R B$ methylation status, $R B$ and TGF- $\beta 1$ protein expression in EBVaGC and matched EBVnGC. The aim of the study is to understand the relationship among EBV, $R B$ and TGF- $\beta 1$ and their role in gastric carcinoma tumorgenesis.

\section{Materials and Methods}

2.1. Patients and Tissue Samples. Fresh and paraffinembedded gastric carcinoma tissues were obtained from 1678 gastric carcinoma patients in Shangdong Province,
China from 2001 to 2009. The positivity of EBV in GC tissues was determined by EBV-encoded small RNA 1 in situ hybridization, as described previously [25]. The clinical features (gender, age, pathologic grade, location, invasion and lymph node metastasis) matched 30 EBVaGC and 38 EBVnGC samples were chosen for study. The study was approved by the Medical Ethics Committee at the Medical College of Qingdao University, China, and informed consent was received from all patients.

2.2. DNA Extraction. DNA was extracted from fresh tumor tissues using the standard method with proteinase $\mathrm{K}$ digestion and phenol-chloroform purification. The QIAamp DNAFFPE Tissue kit (QIAGEN GmbH, Hilden, Germany) was used to extract the DNA from paraffin-embedded tumor tissues.

2.3. Immunohistochemistry (IHC). Paraffin sections were deparaffinized and hydrated as per routine. Rabbit antihuman polyclonal antibody TGF- $\beta 1$ and mouse antihuman monoclonal antibody $R B$ (ZSGB-Bio) were diluted to $1: 50$. The reagents (PV9000 and DAB) were obtained from ZSGBBio and staining was performed as per protocol. PBS (phosphate buffer saline) was used in replacement of primary antibody as a blank control. The section was considered as expressing the protein if cellular staining $\geq 5 \%$, following the methods described previously [26, 27].

2.4. Bisulfite Treatment of Genomic DNA and MethylationSpecific PCR (MSP). $5 \mu \mathrm{g}$ DNA was denatured in $33.3 \mu \mathrm{L}$ of $0.3 \mathrm{~mol} / \mathrm{L} \mathrm{NaOH}$ at $37^{\circ} \mathrm{C}$ for 15 minutes. Denatured DNA was mixed directly with $333 \mu \mathrm{L}$ of bisulfite solution and treated in darkness. The bisulfite solution was prepared as either $2.4 \mathrm{~mol} / \mathrm{L}$ sodium metabisulfite $(\mathrm{pH}$ 5.0-5.2) (Sigma S-1516, St. Louis, MO, USA) $/ 0.5 \mathrm{mmol} / \mathrm{L}$ hydroquinone (Sigma H-7148) for a 4-hour treatment [28]. DNA was desalted and purified using the QIAEX Gel Extraction system (QIAGEN, Cat. no.20021). DNA was then treated with $0.3 \mathrm{~mol} / \mathrm{L} \mathrm{NaOH}$ at $37^{\circ} \mathrm{C}$ for 15 minutes and precipitated with $3 \mathrm{~mol} / \mathrm{L}$ ammonium acetate $(\mathrm{pH} 7.0)$ and $1 \mathrm{~mol} / \mathrm{L}$ sodium acetate ( $\mathrm{pH}$ 5.2). Recovered DNA was dissolved in $100 \mu \mathrm{L}$ of TE buffer ( $\mathrm{pH} \mathrm{8.0)}$ and stored at $-20^{\circ} \mathrm{C}$.

$R B$ promoter methylation status was determined using MSP. In this method, bisulfite treatment converts unmethylated cytosine to uracil, but does not affect the methylated cytosine. Thus, PCR primers can be designed that anneal selectively to methylated or unmethylated DNA after bisulfite conversion. The sequences of the unmethylated DNA and methylated DNA-specific primers are listed in Table 1. The primer UF/UR pair was designed specifically for amplification of the bisulfite-converted unmethylated promoter, while the MF/MR primer pair was designed specifically for the amplification of the bisulfite-converted methylated promoter. MSP results determined whether the samples are methylated or unmethylated. If there is $\mathrm{M}$ primers amplified band, the sample was considered to be in the methylation status. One microliter of bisulfite-treated DNA (around $25 \mathrm{ng}$ ) was amplified with $1.5 \mathrm{mmol} / \mathrm{L} \mathrm{MgCl}_{2}$ and 
TABLE 1: List of primers used in MSP.

\begin{tabular}{|c|c|c|c|c|}
\hline Primers & Sequence & Product size (bp) & Annealing temp $\left(0^{\circ} \mathrm{C}\right)$ & Genomic position \\
\hline$R B M F$ & 5'GGGAGTTTCGCGGACGTGAC3' & 163 & 60 & -61 to 102 \\
\hline$R B M R$ & 5'ACGTCGAAACACGCCCCG3' & & & \\
\hline RBUF & 5'GGGAGTTTTGTGGATGTGAT3' & 163 & 58 & -61 to 102 \\
\hline RBUR & 5' ACATCAAAACACACCCСA3' & & & \\
\hline
\end{tabular}

$0.2 \mathrm{mmol} / \mathrm{L} \mathrm{dNTP}$ in a $25 \mu \mathrm{L}$ reaction volume. Primers were used at a final concentration of $0.4 \mathrm{mmol} / \mathrm{L}$ each. The PCR involved an initial denaturation at $94^{\circ} \mathrm{C}$ for 10 minutes, followed by 40 cycles consisting of $94^{\circ} \mathrm{C}$ for 30 seconds, predetermined optimal annealing temperature for 30 seconds, $72^{\circ} \mathrm{C}$ for 30 seconds, and a final extension at $72^{\circ} \mathrm{C}$ for 5 minutes. Eight microliters of PCR product were analyzed on a $2.0 \%$ agarose gel. Water was used as a negative control.

2.5. Statistical Analysis. $R B$ promoter methylation status, $R B$ expression and TGF- $\beta 1$ expression between EBVaGC and EBVnGC was compared using the Chi-square test. The correlation between promoter methylation and the protein expression was analyzed by Paired fourfold table Chi-square test. The association of clinical features with $R B$ promoter methylation, $R B$ expression, and TGF- $\beta 1$ expression was compared by chi-square test. $P<0.05$ was considered to be statistically significant.

\section{Results}

3.1. Comparison of Clinicopathological Data between EBVaGC and EBVnGC Patients. 102 of 1678 (6.1\%) cases of gastric carcinoma were EBV positive. $30 \mathrm{EBVaGC}$ and $38 \mathrm{EBVnGC}$ tumor tissues with matching clinical parameters were chosen for methylation detection. The clinical and pathological data are listed in Table 2. The two kinds of gastric carcinomas were similar in gender, age, pathologic grade, location, invasion, and lymph node metastasis.

3.2. The Promoter Methylation Status of RB Gene in EBVaGC and $E B V n G C$. Promoter methylation of the $R B$ gene was detected by MSP (Figure 1(a)). In total, 43/68 (63.2\%) cases of gastric carcinomas demonstrated $R B$ gene promoter methylation. The difference in the percent of positive methylation bands detected by MSP for $R B$ was statistically different between EBVaGC (24/30, 80\%) and EBVnGC $(19 / 38,50.0 \%)(P=0.011)$.

The association of clinicopathological parameters of 68 cases with $R B$ gene methylation status was studied. There was no relationship of $R B$ gene methylation status with patient age, gender, pathologic types, and tumor location. However, the methylation status was associated with the depth of tumor invasion $(P=0.036)$ and lymph node metastasis $(P=0.012)$, (Table 3$)$.

3.3. The Promoter Methylation Status of RB Gene in GC and Corresponding Adjacent Normal Gastric Tissues. The
TABle 2: Comparison of clinicopathological data between EBVaGC and EBVnGC patients.

\begin{tabular}{|c|c|c|c|c|}
\hline & $\begin{array}{l}\text { EBVaGC } \\
(n=30)\end{array}$ & $\begin{array}{l}\text { EBVnGC } \\
(n=38)\end{array}$ & $\chi^{2}$ & $P$ \\
\hline \multicolumn{5}{|l|}{ Age (yr) } \\
\hline$<50$ & 18 & 19 & \multirow{2}{*}{0.676} & \multirow{2}{*}{0.411} \\
\hline$\geq 50$ & 12 & 19 & & \\
\hline \multicolumn{5}{|l|}{ Gender } \\
\hline Male & 27 & 31 & \multirow{2}{*}{-} & \multirow{2}{*}{0.494} \\
\hline Female & 3 & 7 & & \\
\hline \multicolumn{5}{|l|}{ Pathologic grade } \\
\hline Poorly differentiated & 28 & 32 & \multirow{2}{*}{-} & \multirow{2}{*}{0.288} \\
\hline $\begin{array}{l}\text { Well-moderately } \\
\text { differentiated }\end{array}$ & 2 & 6 & & \\
\hline \multicolumn{5}{|l|}{ Location } \\
\hline Gastric cardia & 7 & 7 & \multirow{3}{*}{-} & \multirow{3}{*}{0.738} \\
\hline Gastric body & 13 & 15 & & \\
\hline Antrum & 10 & 16 & & \\
\hline \multicolumn{5}{|l|}{ Depth of invasion } \\
\hline $\begin{array}{l}\text { Invasion to serosa and } \\
\text { invasion through serosa }\end{array}$ & 22 & 24 & \multirow[t]{2}{*}{0.793} & \multirow[t]{2}{*}{0.373} \\
\hline Not invading serosa & 8 & 14 & & \\
\hline \multicolumn{5}{|l|}{ Lymph node metastasis } \\
\hline Positive & 17 & 21 & \multirow{2}{*}{0.134} & \multirow{2}{*}{0.908} \\
\hline Negative & 13 & 17 & & \\
\hline
\end{tabular}

promoter methylation of $R B$ gene was detected in EBVaGC and EBVnGC corresponding adjacent normal gastric tissues (Figure 1(b)). The percent of positive methylation bands by MSP for $R B$ in gastric carcinoma and corresponding adjacent normal gastric tissues was $63.2 \%(43 / 68)$ and $39.7 \%(27 / 68)$; the difference was significant $(P=0.006)$.

3.4. The Protein Expression of $R B$ and TGF- $\beta 1 . R B$ and TGF- $\beta 1$ protein expression was detected by IHC, shown in Figures 2(a) and 2(b). The percent of EBVaGC that were positive by IHC for $R B$ was $43.3 \%$ (13/30), lower than in EBVnGC $(63.2 \%, 24 / 38)$, but not significantly different $(P=$ $0.103)$. There was not obvious difference of TGF- $\beta 1$ protein expression between EBVaGC $(56.7 \%, 17 / 30)$ and EBVnGC $(63.2 \%, 24 / 38)(P=0.587)$ (Table 4$)$.

The correlation of $R B$ protein expression with $R B$ promoter methylation was studied. There were 23 negative 


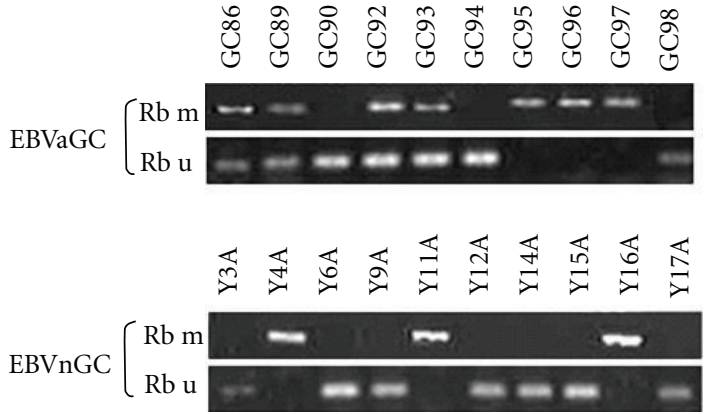

(a)

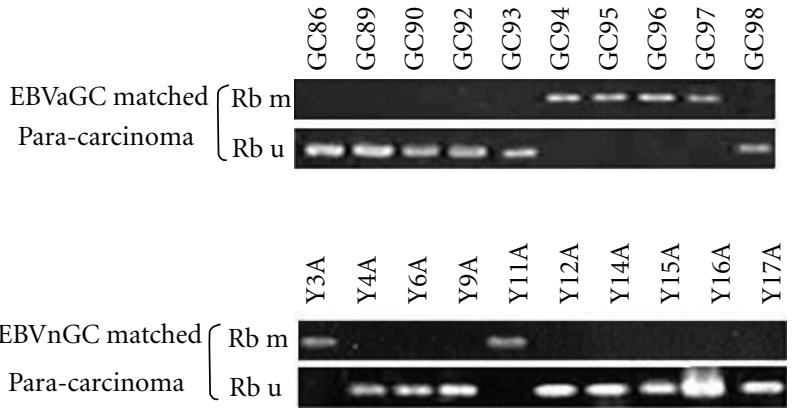

(b)

Figure 1: $R B$ promoter methylation in EBVaGC, EBVnGC, and matched paracarcinoma tissues. (a) Representative $R B$ promoter methylation in EBVaGC and EBVnGC by MSP. U, PCR product from the MSP assay using primers specific for the unmethylated allele; M, PCR product from the MSP assay using primers specific for the methylated allele. (b) Representative RB MSP assay results for matched paracarcinoma tissues.

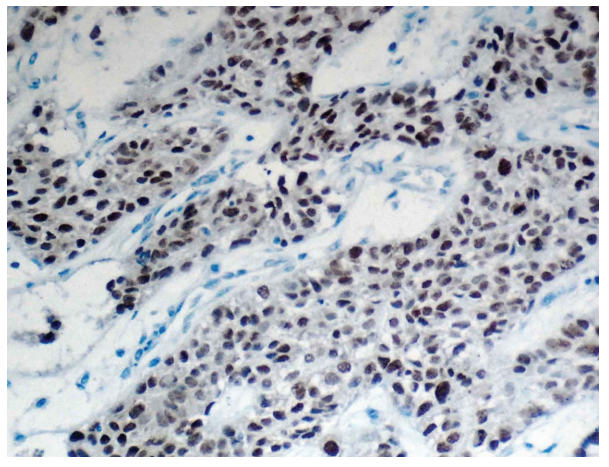

(a)

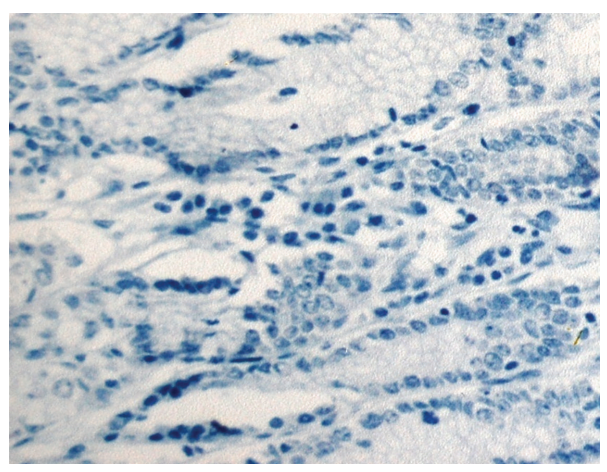

(c)

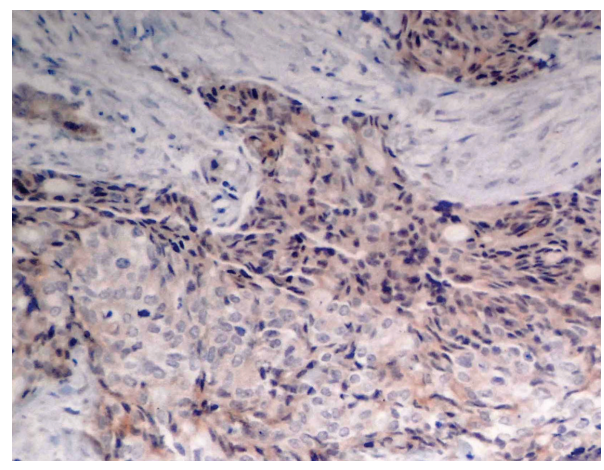

(b)

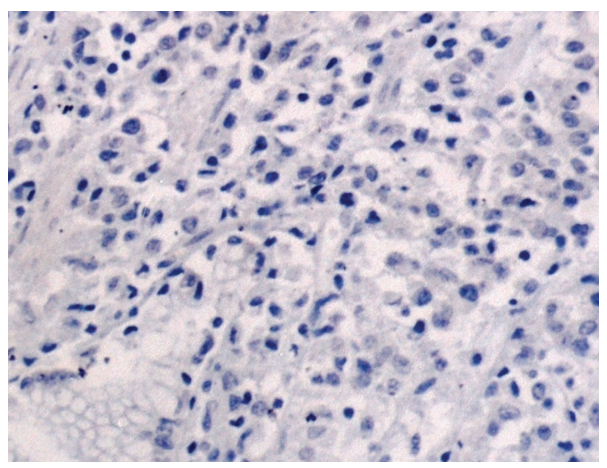

(d)

Figure 2: The protein expression of $R B$ and TGF- $\beta 1$ by immunohistochemistry (magnification $\times 100$ ). (a) Positive immunohistochemistry result of $R B$ in paraffin section. Expression of $R B$ was found in nuclei of gastric carcinoma cells. (b) Positive immunohistochemistry result of TGF- $\beta 1$ in paraffin section. Expression of TGF- $\beta 1$ was found in cytoplasm of gastric carcinoma cells. (c) Negative immunohistochemistry result of $R B$ in paraffin section. (d) Negative immunohistochemistry result of TGF- $\beta 1$ in paraffin section.

$R B$ protein expression cases in $43 R B$ gene methylated gastric carcinoma $(53.5 \%)$, which was higher than in $R B$ gene unmethylated gastric carcinoma $(32.0 \%, 8 / 25)$, but without reverse correlation $(P=0.09, r=0.21)$, (Table 5$)$.

The association between $R B$ expression and clinicopathological parameters is shown in Table 6. There was no relationship between $R B$ protein expression and patients' age, gender, pathological grade, and tumor location, but it was related with the depth of tumor invasion $(P=0.04)$ and lymph node metastasis $(P=0.02)$.

The association between TGF- $\beta 1$ expression and clinicopathological parameters is shown in Table 6. There was 
TABLE 3: Correlation of methylation status of $R B$ gene with clinicopathological data of gastric carcinoma patients.

\begin{tabular}{|c|c|c|c|c|c|}
\hline & $n$ & Methylated $(n)$ & Unmethylated $(n)$ & $\chi^{2}$ & $P$ \\
\hline \multicolumn{6}{|l|}{ EBV infection } \\
\hline EBVaGC & 30 & 24 & 6 & \multirow{2}{*}{6.490} & \multirow{2}{*}{0.011} \\
\hline EBVnGC & 38 & 19 & 19 & & \\
\hline \multicolumn{6}{|l|}{ Age $(y r)$} \\
\hline$<50$ & 37 & 22 & 15 & \multirow{2}{*}{0.498} & \multirow{2}{*}{0.481} \\
\hline$\geq 50$ & 31 & 21 & 10 & & \\
\hline \multicolumn{6}{|l|}{ Gender } \\
\hline Male & 58 & 36 & 22 & \multirow{2}{*}{-} & \multirow{2}{*}{0.835} \\
\hline Female & 10 & 7 & 3 & & \\
\hline \multicolumn{6}{|l|}{ Pathologic grade } \\
\hline Poorly differentiated & 60 & 40 & 20 & \multirow{2}{*}{-} & \multirow{2}{*}{0.216} \\
\hline Well-moderately differentiated & 8 & 3 & 5 & & \\
\hline \multicolumn{6}{|l|}{ Location } \\
\hline Gastric cardia & 14 & 10 & 4 & \multirow{3}{*}{3.172} & \multirow{3}{*}{0.205} \\
\hline Gastric body & 28 & 20 & 8 & & \\
\hline Antrum & 26 & 13 & 13 & & \\
\hline \multicolumn{6}{|l|}{ Depth of invasion } \\
\hline Invasion to serosa and invasion through serosa & 46 & 33 & 13 & \multirow{2}{*}{4.423} & \multirow{2}{*}{0.036} \\
\hline Not invading serosa & 22 & 10 & 12 & & \\
\hline \multicolumn{6}{|l|}{ Lymph node metastasis } \\
\hline Positive & 38 & 29 & 9 & \multirow{2}{*}{6.339} & \multirow{2}{*}{0.012} \\
\hline Negative & 30 & 14 & 16 & & \\
\hline
\end{tabular}

TABLE 4: Comparisons of the expression of $R B$ and TGF- $\beta 1$ between EBVaGC and EBVnGC.

\begin{tabular}{lccccc}
\hline & \multirow{2}{*}{$n$} & \multicolumn{2}{c}{$R B$ expression } & \multicolumn{2}{c}{ TGF- $\beta 1$ expression } \\
& & Positive & Negative & Positive & Negative \\
\hline EBVaGC & 30 & 13 & 17 & 17 & 13 \\
EBVnGC & 38 & 24 & 14 & 24 & 14 \\
$\chi^{2}$ & & \multicolumn{2}{c}{2.656} & & 0.295 \\
$P$ & & \multicolumn{2}{c}{0.103} & \multicolumn{2}{c}{0.587} \\
\hline
\end{tabular}

TABLE 5: Correlation of methylation status of $R B$ gene with its protein expression.

\begin{tabular}{lccc}
\hline \multirow{2}{*}{ Methylation status } & \multicolumn{2}{c}{ Protein expression } & \multirow{2}{*}{ Total } \\
& Positive & Negative & \\
\hline Methylated & 20 & 23 & 43 \\
Unmethylated & 17 & 8 & 25 \\
\hline Total & 37 & 31 & 68 \\
\hline
\end{tabular}

no relationship between $T G F-\beta 1$ protein expression and patients' age, gender, pathological types, and tumor location, but there was positive association between TGF- $\beta 1$ protein expression and the depth of tumor invasion $(P=0.02)$ and lymph node metastasis $(P=0.002)$.

\section{Discussion}

In this study, the percent of EBVaGC with positive methylation bands by MSP for $R B$ was significantly higher than that of EBVnGC, indicating that EBV may induce $R B$ promoter methylation during infection. Previous studies showed that EBVaGC had higher methylation frequency and promoter CpGI methylation density than EBVnGC in some TSGs, such as p16, E-cadherin and p73, and the methylation status was reverse correlated with protein expression $[4,10,29]$. These results indicate that methylation and silence of TSGs induced by EBV may be an important oncogenic mechanism for the development of EBVaGC. Only a few studies detected the methylation of $R B$ in gastric carcinomas. Zhao et al. [30] found that the percent of positive methylation bands for $R B$ gene was $44.6 \%$ (45/101), similar to our study of 38 EBVnGC (50\%), but less than that of $30 \mathrm{EBVaGC}$ cases $(80 \%)$, which provides further support that EBV induces $R B$ gene methylation in EBVaGC.

Promoter $\mathrm{CpG}$ island methylation is considered an important mechanism of TSG inactivation. In the present study, 23 of $43(53.5 \%)$ methylated gastric carcinoma tissues lost $R B$ protein expression, which was higher than that in unmethylated gastric carcinoma tissues $(32.0 \%, 8 / 25)$, but $R B$ promoter methylation was not reversely correlated with $R B$ protein expression $(P=0.086)$. This phenomenon was also found between p16INK4 gene methylation status and expression in meningiomas by Tse et al. [31]. The possible explanations include: (1) gene methylation in gastric carcinoma tissue is heterogeneous; (2) gene methylation may occur in only one allele of cancer cells, while the other allele remains unmethylated. The above reasons may also explain the existence of methylated and unmethylated gene 
TABLE 6: Correlation of expression of $R B$ and TGF- $\beta 1$ protein with clinicopathological data of gastric carcinoma patients.

\begin{tabular}{|c|c|c|c|c|c|c|c|c|c|}
\hline & \multirow{2}{*}{$n$} & \multicolumn{4}{|c|}{$R B$} & \multicolumn{4}{|c|}{$T G F-\beta 1$} \\
\hline & & Expression $(n)$ & Absent $(n)$ & $\chi^{2}$ & $P$ & Expression $(n)$ & Absent $(n)$ & $\chi^{2}$ & $P$ \\
\hline \multicolumn{10}{|l|}{ Age (yr) } \\
\hline$<50$ & 37 & 21 & 16 & \multirow{2}{*}{0.180} & \multirow{2}{*}{0.671} & 20 & 17 & \multirow{2}{*}{1.320} & \multirow{2}{*}{0.251} \\
\hline$\geq 50$ & 31 & 16 & 15 & & & 21 & 10 & & \\
\hline \multicolumn{10}{|l|}{ Gender } \\
\hline Male & 58 & 31 & 27 & \multirow{2}{*}{-} & \multirow{2}{*}{0.972} & 35 & 23 & \multirow{2}{*}{-} & \multirow{2}{*}{0.742} \\
\hline Female & 10 & 6 & 4 & & & 6 & 4 & & \\
\hline \multicolumn{10}{|l|}{ Pathologic grade } \\
\hline Poorly differentiated & 60 & 31 & 29 & \multirow{2}{*}{-} & \multirow{2}{*}{0.428} & 38 & 22 & \multirow{2}{*}{-} & \multirow{2}{*}{0.295} \\
\hline Well-moderately differentiated & 8 & 6 & 2 & & & 3 & 5 & & \\
\hline \multicolumn{10}{|l|}{ Location } \\
\hline Gastric cardia & 14 & 7 & 7 & \multirow{3}{*}{2.091} & \multirow{3}{*}{0.352} & 8 & 6 & \multirow{3}{*}{0.318} & \multirow{3}{*}{0.853} \\
\hline Gastric body & 28 & 13 & 15 & & & 18 & 10 & & \\
\hline Antrum & 26 & 17 & 9 & & & 15 & 11 & & \\
\hline \multicolumn{10}{|l|}{ Depth of invasion } \\
\hline Invasion to serosa and invasion through serosa & 46 & 21 & 25 & \multirow{2}{*}{4.398} & \multirow{2}{*}{0.036} & 32 & 14 & \multirow{2}{*}{5.105} & \multirow{2}{*}{0.024} \\
\hline Not invading serosa & 22 & 16 & 6 & & & 9 & 13 & & \\
\hline \multicolumn{10}{|l|}{ Lymph node metastasis } \\
\hline Positive & 38 & 16 & 22 & \multirow{2}{*}{5.259} & \multirow{2}{*}{0.022} & 29 & 9 & \multirow{2}{*}{9.235} & \multirow{2}{*}{0.002} \\
\hline Negative & 30 & 21 & 9 & & & 12 & 18 & & \\
\hline
\end{tabular}

bands by MSP. Increasing of CpG island methylation density is a dynamic process, and only the methylation density increases to a certain extent, it results in the complete loss of the expression. The $R B$ promotor methylation could result the decrease or loss of protein expression. Thus, lacking reverse correlation between $R B$ promotor methylation and protein expression was not contradictory. Because of the limitation of major disadvantage of MSP, the methylation status of single $\mathrm{CpG}$ site in primer binding sequences is not be detected [32]. The correlation between $R B$ promoter methylation dynamic change and protein expression need further study. These reasons above can also be used to explain why there wasn't a significant difference in $R B$ protein expression between EBVaGC and EBVnGC, even though $R B$ promoter methylation of EBVaGC was significantly higher than EBVnGC. If the $R B$ gene promoter methylation and its protein expression were negatively correlated, $R B$ protein expression in EBVaGC should have been significantly lower than that in EBVnGC. In this study, the percent of EBVaGC and EBVnGC that were positive by IHC for $R B$ were $43.3 \%$ and $63.2 \%$, respectively. Although no significant difference was found of the positive rate of $R B$ protein expression between EBVaGC and EBVnGC, the relatively lower expression rate in EBVaGC also suggests that EBVinduced $R B$ promoter methylation could lead to inhibition of $R B$ protein expression to some extent. Moreover, the promoter methylation of $R B$ gene was also detected in GC and corresponding adjacent normal gastric tissues and the difference was significant, which confirmed that RB promoter methylation is involved with the development of GC.
Similar to the result of $R B$ protein expression, the positive rate of TGF- $\beta 1$ protein expression was $56.7 \%(17 / 30)$ and $63.2 \%(24 / 38)$ in EBVaGC and EBVnGC, respectively, without significant difference $(P=0.404)$, suggesting that EBV is not related to the TGF- $\beta 1$ expression in EBVaGC. Kim et al. [33] examined the association of EBV with $R B$ and p53 protein expression in classic Hodgkin lymphoma and found that EBV wasn't associated with $R B$ and $p 53$ protein expression. $\mathrm{Xu}$ et al. [34] found TGF- $\beta 1$ level in the serum of nasopharyngeal cancer patients was significantly higher than that in normal persons, and also the advanced stage was higher than the early stage, and recurrent tumors was higher than primary tumors, which indicating that serum TGF- $\beta 1$ can be used for diagnosis and judgment for prognosis of NPC, and EBV infection can induce the synthesis and release of TGF- $\beta 1$. This result was different from our result of TGF$\beta 1$ in EBVaGC.

Previous studies showed that TGF- $\beta 1$ expression rate and expression level were higher in gastric carcinoma tissues than that in normal tissues, and the TGF- $\beta 1$ expression were associated with gastric invasion, metastases, and prognosis [35-37]. In the advanced cancer, TGF- $\beta 1$ can provide the microenvironment suitable for tumor growth, invasion, and metastases by stimulating angiogenesis, cell spread, immune suppression, and synthesis of extracellular matrix. In gastric carcinoma, $R B$ protein loss was also found to be associated with metastases and prognosis [38-41]. The $R B$ protein expression rate was $40 \% \sim 90 \%[38-40,42,43]$ and $T G F-$ $\beta 1$ protein expression rate was $22.8 \% \sim 71 \%$ [35-37, 44] in previous studies. In the present study, $R B$ protein loss and $T G F-\beta 1$ protein wasn't associated with patient age, gender, 
pathologic types, and tumor location, but associated with the depth of tumor invasion and lymph node metastasis. At the same time, we also confirmed that $R B$ promoter methylation was associated with tumor invasion and lymph node metastasis, indicating that $R B$ promoter methylation, $R B$ and $T G F-\beta 1$ protein expression can be as clinical reference index for judgement of gastric carcinoma invasion and metastasis.

\section{Conclusion}

Our study showed that Aberrant $R B$ promoter methylation was common in gastric carcinoma. EBV could induce $R B$ gene methylation and affect the gene expression in EBVaGC development. EBV has no significant effect on TGF- $\beta 1$ expression.

\section{Abbreviations}

$\begin{array}{ll}\text { GC: } & \text { Gastric carcinoma } \\ \text { EBV: } & \text { Epstein-Barr virus } \\ \text { EBVaGC: } & \text { Epstein-Barr virus-associated gastric } \\ & \text { carcinoma } \\ \text { EBVnGC: } & \text { EBV-negative gastric carcinoma } \\ \text { TSG: } & \text { Tumor suppressor gene } \\ R B: & \text { Retinoblastoma } \\ T G F-\beta 1: & \text { Transforming growth factor- } \beta 1 .\end{array}$

\section{Conflict of Interests}

None of the authors has any conflicts of interests.

\section{Acknowledgments}

This research was supported by the Grant from National Natural Science Foundation of China (NSFC 30970157). The authors thank Stacey Barron (University of Pittsburgh, Pittsburgh, PA, USA) for her helpful review of the paper.

\section{References}

[1] P. Correa, M. B. Piazuelo, and M. C. Camargo, "The future of gastric cancer prevention," Gastric Cancer, vol. 7, no. 1, pp. 9-16, 2004.

[2] H. Uozaki and M. Fukayama, "Epstein-Barr virus and gastric carcinoma-viral carcinogenesis through epigenetic mechanisms," International Journal of Clinical and Experimental Pathology, vol. 1, no. 3, pp. 198-216, 2008.

[3] S. Imai, S. Koizumi, M. Sugiura et al., "Gastric carcinoma: monoclonal epithelial malignant cells expressing Epstein-Barr virus latent infection protein," Proceedings of the National Academy of Sciences of the United States of America, vol. 91, no. 19, pp. 9131-9135, 1994.

[4] T. Ushiku, J. M. Chong, H. Uozaki et al., "p73 gene promoter methylation in Epstein-Barr virus-associated gastric carcinoma," International Journal of Cancer, vol. 120, no. 1, pp. 60-66, 2007.
[5] S. Akiba, C. Koriyama, R. Herrera-Goepfert, and Y. Eizuru, "Epstein-Barr virus associated gastric carcinoma: epidemiological and clinicopathological features," Cancer Science, vol. 99, no. 2, pp. 195-201, 2008.

[6] J. H. Lee, S. H. Kim, S. H. Han, J. S. An, E. S. Lee, and Y. S. Kim, "Clinicopathological and molecular characteristics of Epstein-Barr virus-associated gastric carcinoma: a metaanalysis," Journal of Gastroenterology and Hepatology, vol. 24, no. 3, pp. 354-365, 2009.

[7] M. Fukayama, R. Hino, and H. Uozaki, "Epstein-Barr virus and gastric carcinoma: virus-host interactions leading to carcinoma," Cancer Science, vol. 99, no. 9, pp. 1726-1733, 2008.

[8] R. Taby and J. P. Issa, "Cancer epigenetics," Cancer Journal for Clinicians, vol. 60, no. 6, pp. 376-392, 2010.

[9] K. Sakuma, J. M. Chong, M. Sudo et al., "High-density methylation of p14ARF and P16INK4A in Epstein-Barr virusassociated gastric carcinoma," International Journal of Cancer, vol. 112, no. 2, pp. 273-278, 2004.

[10] M. Sudo, J. M. Chong, K. Sakuma et al., "Promoter hypermethylation of E-cadherin and its abnormal expression in Epstein-Barr virus-associated gastric carcinoma," International Journal of Cancer, vol. 109, no. 2, pp. 194-199, 2004.

[11] G. H. Kang, S. Lee, W. H. Kim et al., "Epstein-Barr viruspositive gastric carcinoma demonstrates frequent aberrant methylation of multiple genes and constitutes $\mathrm{CpG}$ island methylator phenotype-positive gastric carcinoma," American Journal of Pathology, vol. 160, no. 3, pp. 787-794, 2002.

[12] K. G. Wiman, "The retinoblastoma gene: role in cell cycle control and cell differentiation," FASEB Journal, vol. 7, no. 10, pp. 841-845, 1993.

[13] R. J. Akhurst and R. Derynck, "TGF- $\beta$ signaling in cancer-a double-edged sword," Trends in Cell Biology, vol. 11, no. 11, pp. S44-S51, 2001.

[14] L. Attisano and J. L. Wrana, "Signal transduction by the TGF- $\beta$ superfamily," Science, vol. 296, no. 5573, pp. 1646-1647, 2002.

[15] M. Lutz and P. Knaus, "Integration of the TGF- $\beta$ pathway into the cellular signalling network," Cellular Signalling, vol. 14, no. 12, pp. 977-988, 2002.

[16] S. S. Huang, S. M. Leal, C. L. Chen, I. H. Liu, and J. S. Huang, "Cellular growth inhibition by TGF-betal involves IRS proteins," FEBS Letters, vol. 565, no. 1-3, pp. 117-121, 2004.

[17] M. Fukuda, W. Kurosaki, K. Yanagihara, H. Kuratsune, and T. Sairenji, "A mechanism in epstein-barr virus oncogenesis: inhibition of transforming growth factor- $\beta 1$-mediated induction of MAPK/p21 by LMP1," Virology, vol. 302, no. 2, pp. 310-320, 2002.

[18] M. Fukuda, K. Ikuta, K. Yanagihara et al., "Effect of transforming growth factor- $\beta 1$ on the cell growth and Epstein-Barr virus reactivation in EBV-infected epithelial cell lines," Virology, vol. 288, no. 1, pp. 109-118, 2001.

[19] S. Imai, S. Koizumi, M. Sugiura et al., "Gastric carcinoma: monoclonal epithelial malignant cells expressing Epstein-Barr virus latent infection protein," Proceedings of the National Academy of Sciences of the United States of America, vol. 91, no. 19, pp. 9131-9135, 1994.

[20] L. Cito, F. Pentimalli, I. Forte, E. Mattioli, and A. Giordano, "Rb family proteins in gastric cancer," Oncology Reports, vol. 24, no. 6, pp. 1411-1418, 2010.

[21] J. Ananiev, M. Gulubova, G. Tchernev et al., "Relation between transforming growth factor-betal expression, its receptor and clinicopathological factors and survival in HER2-negative gastric cancers," Wiener Klinische Wochenschrift, vol. 123, no. 21-22, pp. 668-673, 2011. 
[22] S. A. Galetsky, V. V. Tsvetnov, C. E. Land et al., "EpsteinBarr-Virus-associated gastric cancer in Russia," International Journal of Cancer, vol. 73, no. 6, pp. 786-789, 1997.

[23] M. S. Chang, H. S. Lee, C. W. Kim, Y. I. Kim, and W. H. Kim, "Clinicopathologic characteristics of Epstein-Barr virusincorporated gastric cancers in Korea," Pathology Research and Practice, vol. 197, no. 6, pp. 395-400, 2001.

[24] P. Mukherjee, S. L. Winter, and M. G. Alexandrow, "Cell cycle arrest by transforming growth factor $\beta 1$ near G $1 / \mathrm{S}$ is mediated by acute abrogation of prereplication complex activation involving an Rb-MCM interaction," Molecular and Cellular Biology, vol. 30, no. 3, pp. 845-856, 2010.

[25] Y. Wang, K. Kanai, Y. Satoh, B. Luo, and T. Sairenji, "Carboxylterminal sequence variation of latent membrane protein 1 gene in Epstein-Barr virus-associated gastric carcinomas from eastern China and Japan," Intervirology, vol. 50, no. 3, pp. 229236, 2007.

[26] H. M. Khaled, A. A. Bahnassy, A. A. Raafat, A. R. N. Zekri, M. S. Madboul, and N. M. Mokhtar, "Clinical significance of altered nm23-H1, EGFR, RB and p53 expression in bilharzial bladder cancer," BMC Cancer, vol. 9, p. 32, 2009.

[27] A. Valkov, S. W. Sorbye, T. K. Kilvaer et al., "The prognostic impact of TGF- $\beta 1$, fascin, NF- $\kappa \mathrm{b}$ and PKC- $\zeta$ expression in soft tissue sarcomas," PLoS ONE, vol. 6, no. 3, Article ID e17507, 2011.

[28] Q. Tao, H. Huang, T. M. Geiman et al., "Defective de novo methylation of viral and cellular DNA sequences in ICF syndrome cells," Human Molecular Genetics, vol. 11, no. 18, pp. 2091-2102, 2002.

[29] T. Osawa, J. M. Chong, M. Sudo et al., "Reduced expression and promoter methylation of p16 gene in Epstein-Barr virusassociated gastric carcinoma," Japanese Journal of Cancer Research, vol. 93, no. 11, pp. 1195-1200, 2002.

[30] Y. F. Zhao, Y. G. Zhang, X. X. Tian, J. Du, and J. Zheng, "Aberrant methylation of multiple genes in gastric carcinomas," International Journal of Surgical Pathology, vol. 15, no. 3, pp. 242-251, 2007.

[31] J. Y. M. Tse, H. K. Ng, K. W. Lo et al., "Analysis of cell cycle regulators: p161NK4A, pRb, and CDK4 in low- and highgrade meningiomas," Human Pathology, vol. 29, no. 11, pp. 1200-1207, 1998.

[32] C. Dahl and P. Guldberg, "DNA methylation analysis techniques," Biogerontology, vol. 4, no. 4, pp. 233-250, 2003.

[33] L. H. Kim, S. C. Peh, and S. Poppema, "Expression of retinoblastoma protein and P16 proteins in classic Hodgkin lymphoma: relationship with expression of p53 and presence of Epstein-Barr virus in the regulation of cell growth and death," Human Pathology, vol. 37, no. 1, pp. 92-100, 2006.

[34] J. Xu, J. Menezes, U. Prasad, and A. Ahmad, "Elevated serum levels of transforming growth factor betal in EpsteinBarr virus-associated nasopharyngeal carcinoma patients," International Journal of Cancer, vol. 8484, no. 4, pp. 396-399, 1999.

[35] K. Vagenas, C. Spyropoulos, V. Gavala, and A. C. Tsamandas, "TGFbeta1, TGFbeta2, and TGFbeta3 protein expression in gastric carcinomas: correlation with prognostics factors and patient survival," Journal of Surgical Research, vol. 139, no. 2, pp. 182-188, 2007.

[36] H. Saito, S. Tsujitani, S. Oka et al., "The expression of transforming growth factor-betal is significantly correlated with the expression of vascular endothelial growth factor and poor prognosis of patients with advanced gastric carcinoma," Cancer, vol. 86, no. 8, pp. 1455-1462, 1999.
[37] Y. Maehara, Y. Kakeji, A. Kabashima et al., "Role of transforming growth factor- $\beta 1$ in invasion and metastasis in gastric carcinoma," Journal of Clinical Oncology, vol. 17, no. 2, pp. 607-614, 1999.

[38] G. Kouraklis, I. E. Katsoulis, S. Theocharis et al., "Does the expression of cyclin E, pRb, and p21 correlate with prognosis in gastric adenocarcinoma?" Digestive Diseases and Sciences, vol. 54, no. 5, pp. 1015-1020, 2009.

[39] H. S. Song, I. H. Kim, S. S. Sohn, K. Y. Kwon, and W. S. Lee, "Prognostic significance of immunohistochemical expression of p53 and retinoblastoma gene protein (pRB) in curatively resected gastric cancer," Korean Journal of Internal Medicine, vol. 20, no. 1, pp. 1-7, 2005.

[40] R. M. Feakins, C. D. Nickols, H. Bidd, and S. J. Walton, "Abnormal expression of pRb, p16, and Cyclin D1 in Gastric adenocarcinoma and its lymph node metastases: relationship with pathological features and survival," Human Pathology, vol. 34, no. 12, pp. 1276-1282, 2003.

[41] N. H. Chou, H. C. Chen, N. S. Chou, P. I. Hsu, and H. H. Tseng, "Expression of altered retinoblastoma protein inversely correlates with tumor invasion in gastric carcinoma," World Journal of Gastroenterology, vol. 12, no. 44, pp. 7188-7191, 2006.

[42] I. Kishimoto, H. Mitomi, Y. Ohkura, H. Kanazawa, N. Fukui, and M. Watanabe, "Abnormal expression of p16INK4a, cyclin D1, cyclin-dependent kinase 4 and retinoblastoma protein in gastric carcinomas," Journal of Surgical Oncology, vol. 98, no. 1, pp. 60-66, 2008.

[43] X. S. He, Y. H. Rong, Q. Su et al., "Expression of p16 gene and $\mathrm{Rb}$ protein in gastric carcinoma and their chinicopathological significance," World Journal of Gastroenterology, vol. 11, no. 15, pp. 2218-2223, 2005.

[44] V. Zolota, A. Batistatou, A. C. Tsamandas, G. Iliopoulos, C. D. Scopa, and D. S. Bonikos, "Immunohistochemical expression of TGF- $\beta 1$, p21WAF1, p53, Ki67, and angiogenesis in gastric carcinomas: a clinicopathologic study," International Journal of Gastrointestinal Cancer, vol. 32, no. 2-3, pp. 83-89, 2002. 


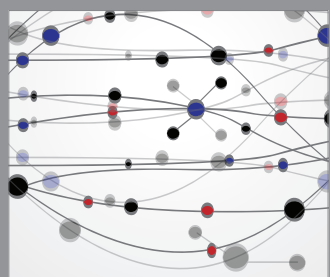

The Scientific World Journal
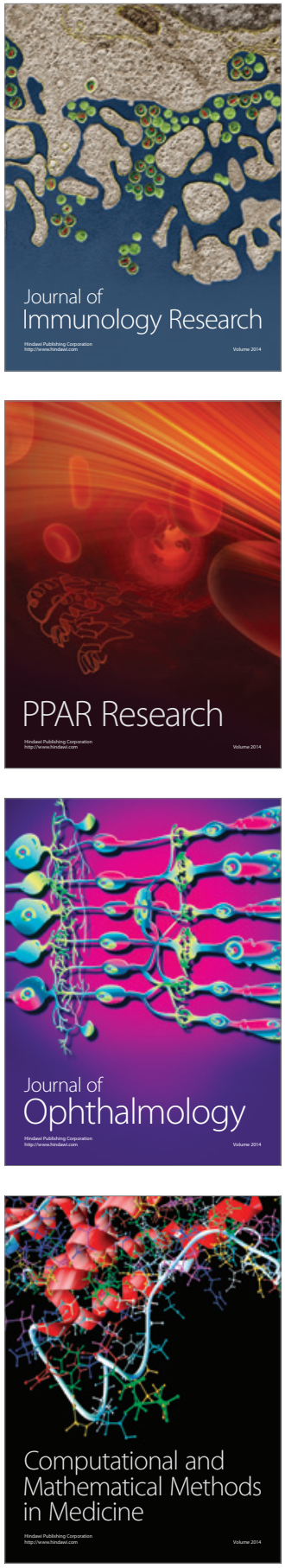

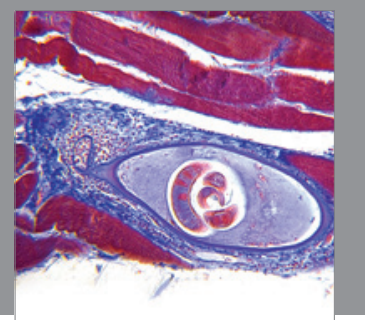

Gastroenterology

Research and Practice
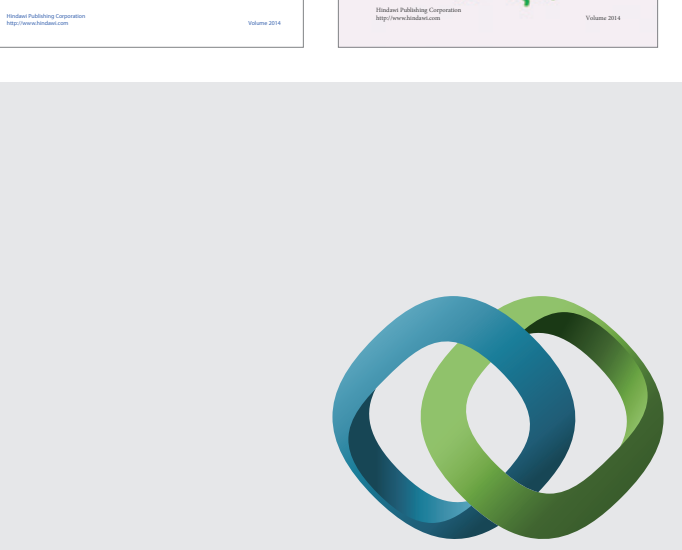

\section{Hindawi}

Submit your manuscripts at

http://www.hindawi.com
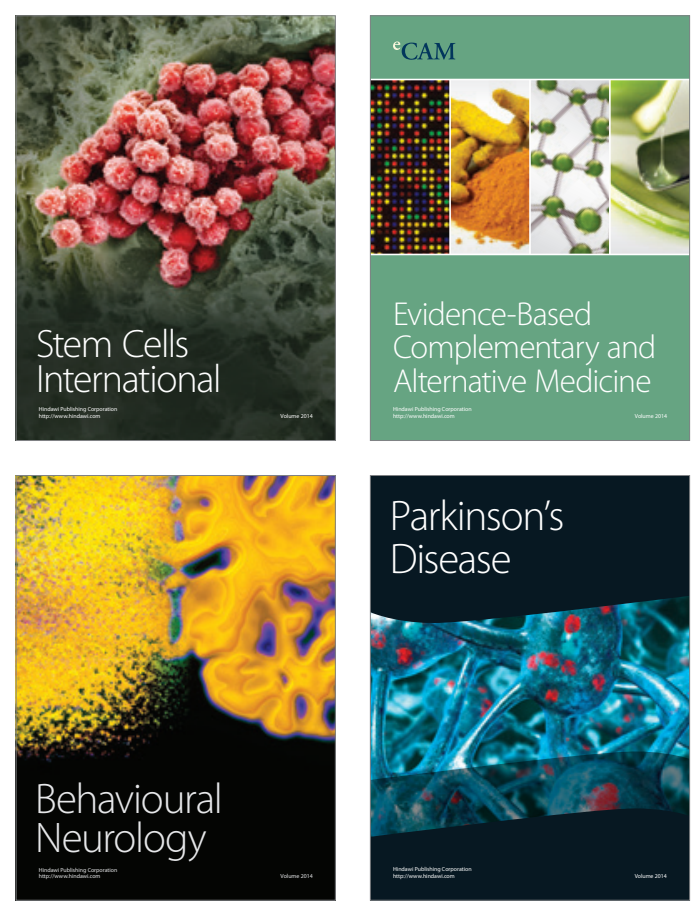

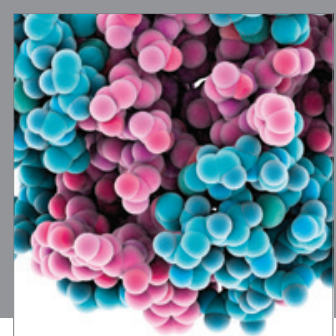

Journal of
Diabetes Research

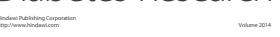

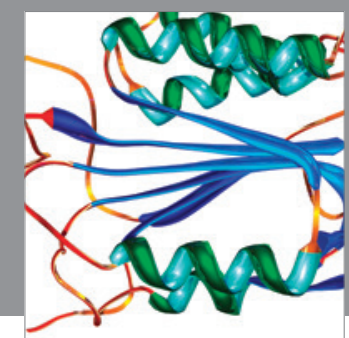

Disease Markers
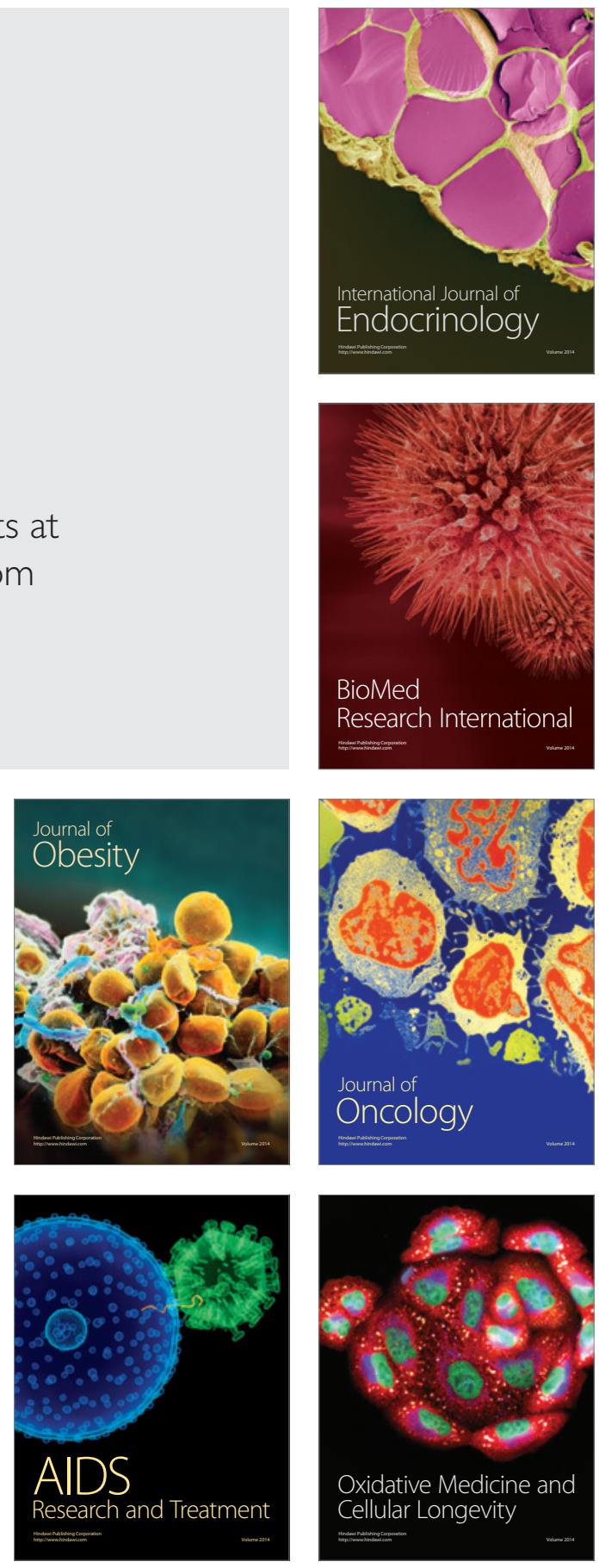